\title{
Racial differences in the responses to shear stress in human umbilical vein endothelial cells
}

\author{
This article was published in the following Dove Press journal: \\ Vascular Health and Risk Management \\ 8 July 2011 \\ Number of times this article has been viewed
}

\author{
Deborah L Feairheller ${ }^{1,4}$ \\ Joon-Young Park ${ }^{2}$ \\ Victor Rizzo 3 \\ Boa Kim ${ }^{2}$ \\ Michael D Brown ${ }^{1,3}$ \\ 'Hypertension, Molecular and \\ Applied Physiology Laboratory, \\ ${ }^{2}$ Cardiovascular Genomics Laboratory, \\ Department of Kinesiology, \\ ${ }^{3}$ Cardiovascular Research Center, \\ School of Medicine, Temple University, \\ Philadelphia, PA, USA; ${ }^{4}$ Exercise \\ and Metabolic Disease Research \\ Laboratory, School of Nursing, \\ University of California Los Angeles, \\ Los Angeles, CA, USA
}

Background: African American ethnicity is an independent risk factor for exaggerated oxidative stress, which is related to inflammation, hypertension, and cardiovascular disease. Recently, we reported that in vitro oxidative stress and inflammation levels differ between African American and Caucasian human umbilical vein endothelial cells (HUVECs), African American HUVECs having higher levels of both. However, it remains to be shown whether the cells would respond differently to external stimuli.

Methods: We used a cone and plate viscometer to apply laminar shear stress (LSS) as an aerobic exercise mimetic to compare the responses by race. HUVECs were exposed to static conditions (no LSS), low LSS (5 dyne/ $\left.\mathrm{cm}^{2}\right)$, and moderate LSS (20 dyne $\left./ \mathrm{cm}^{2}\right)$.

Results: It was found that African American HUVECs had higher levels of oxidative stress under static conditions, and when LSS was applied protein expression levels (NADPH oxidase NOX2, NOX4 and p47phox subunits, eNOS, SOD2, and catalase) and biomarkers (NO, SOD, and total antioxidant capacity) were modulated to similar levels between race.

Conclusion: African American HUVECs may be more responsive to LSS stimulus indicating that aerobic exercise prescriptions may be valuable for this population since the potential exists for large in vivo improvements in oxidative stress levels along the endothelial layer in response to increased shear flow.

Keywords: shear stress, African American, NADPH oxidase, HUVECs, oxidative stress

\section{Introduction}

African Americans exhibit higher incidence of cardiovascular disease (CVD), renal dysfunction, hypertension (HT), and heightened inflammation than other ethnic groups, in particular Caucasians. ${ }^{1,2}$ African Americans also have higher levels of reactive oxygen species (ROS), attenuated antioxidant capacity, and thus favor a pro-oxidant or oxidative stress state. Oxidative stress and inflammation have independently been associated with the pathology and progression of HT and CVD, and compelling evidence indicates that endothelial dysfunction is recognized as the hallmark step in the progression of overt HT. ${ }^{3-5}$ Racial differences have also been reported in the development, progression, and response to CVD, HT, and endothelial dysfunction, yet molecular biology studies aimed at identifying mechanisms neglect to consider the racial origin of cultured cells. Properly understanding and defining potential molecular differences for the in vivo disparity could help in clinical diagnoses as well as potentially guide therapeutic treatments.

Recently we used a parallel cell culture experimental design to compare oxidative stress and inflammation levels between African American and Caucasian human
Correspondence: Michael D Brown I29 Pearson Hall, Broad \& Montgomery Avenue, Philadelphia, PA 19122, USA Phone +I 2152045218

$\mathrm{Fax}+\mathrm{I} 2152044414$

Email brownmd@temple.edu 
umbilical vein endothelial cells (HUVECs). ${ }^{6}$ In that study we found that, under basal conditions when compared with Caucasian HUVECs, African American HUVECs exhibited enhanced oxidative stress and inflammation as indicated by increased protein expression of nicotinamide adenine dinucleotide phosphate (NADPH) oxidase subunits, interleukin-6, inducible nitric oxidase synthase (NOS), and lower superoxide dismutase (SOD) activity. We also found relatively heightened endothelial (eNOS) expression and nitric oxide (NO) end-product production. It remains to be shown whether there would be racial differences in the response to laminar shear stress (LSS) stimulation between cells of African American and Caucasian origin.

Thus the purpose of this observational study was to compare HUVEC responses, by race, to the application of LSS, using a cone and plate viscometer. In parallel, HUVECs from both African Americans and Caucasians were exposed to static conditions (no LSS), to low LSS ( $5 \mathrm{dyne} / \mathrm{cm}^{2}$ ), and to moderate LSS (20 dyne $/ \mathrm{cm}^{2}$ ). As a translational model, LSS was used as an in vitro exercise mimetic. Traditional aerobic exercise causes an increase in blood flow along the vessel walls which is thought to be responsible for reductions in endothelial dysfunction and improvements in oxidative stress levels. It is hoped that the results from this observational study can guide future in vitro mechanistic studies. Furthermore, clinically it is hoped that these results can guide nonpharmacologic lifestyle modifications, like prescriptions of aerobic exercise therapy, by elucidating potential race-dependent differences in endothelial cell responses to increased shear flow.

\section{Methods}

\section{Cell culture}

African American $(\mathrm{N}=3)$ and Caucasian $(\mathrm{N}=3)$ primary culture HUVECs from separate donors were purchased from Lonza (Walkersville, MD), and all experiments were conducted at passage four or five. HUVECs were cultured in parallel in EGM-2 medium supplemented with $2 \%$ fetal bovine serum (FBS) and growth factors (Lonza, Walkersville, MD). For NO measurement, HUVECs were grown in phenol-red free EGM-2 medium because it has been reported that the addition of phenol red to medium could potentially interfere with nitrate and nitrite measurements. ${ }^{7}$ All cells were maintained at $37^{\circ} \mathrm{C}$ in a $5 \% \mathrm{CO}_{2}$ atmosphere, in $100 \mathrm{~mm}$ tissue culture dishes. Application of LSS was done when cells reached log phase and were at 90\%-100\% confluence. All experiments were repeated for confirmation, HUVECs were treated identically, and samples used for all assays were tested in duplicate. All Lonza HUVECs are characterized by morphological observation through serial passaging, positive test for von Willebrand Factor VIII and acetylated low-density lipoprotein uptake, and negative test for $\alpha$-smooth muscle actin.

\section{In vitro flow: LSS}

Confluent HUVECs grown in separate 100-mm tissue culture dishes were exposed in parallel for a period of 24 hours to LSS at two levels: $5 \mathrm{dyne} / \mathrm{cm}^{2}$ and $20 \mathrm{dyne} / \mathrm{cm}^{2}$, using a rotating Teflon cone $\left(0.5^{\circ}\right.$ cone angle $)$ according to the methods of Mowbray et al. ${ }^{8}$ Static controls (no LSS) remained in the incubator for the same 24-hour period.

\section{Protein expression: Western blotting}

Immediately following LSS application, both the static and LSS culture dishes were harvested for protein analysis using methods previously described. ${ }^{6}$ Aliquots of cell lysate were separated by SDS-PAGE on 10\% gels and transferred to PVDF membranes, which were blocked with 5\% nonfat dry milk dissolved in Tris-Buffered saline and then incubated overnight with primary antibodies at $4^{\circ} \mathrm{C}$. Immunoreactive proteins were detected by chemiluminescence with Thermo Scientific SuperSignal (Pierce Biotechnology, IL). Primary antibodies included anti-NOX4 (Pierce Biotechnology, IL), anti-gp91phox (NOX2) (BD Bioscience, CA), anti-p47phox (BD Biosciences, CA), anti-SOD2 (AbFrontier), anti-CAT (Calbiochem, CA), and anti-eNOS (BD Biosciences, CA). The anti- $\beta$-actin antibody (Santa Cruz Biotechnology, CA) was used for internal control.

\section{Oxidative stress measurement}

Following LSS application, cell supernatant was collected for NO measurements from both static and LSS treated HUVECs and immediately stored at $-80^{\circ} \mathrm{C}$ until assay. Cell lysate was collected by fractionation using methods previously described. ${ }^{6}$ NO end-products, SOD, and total antioxidant capacity (TAC) activity were measured as described. ${ }^{9}$ Culture samples were not diluted for any oxidative stress measurement.

\section{Statistical analysis}

Data analysis included descriptive statistics. Within-HUVEC group analysis was performed using paired $t$-tests comparing before and after LSS values at each LSS level. BetweenHUVEC racial group analysis was performed using independent $t$-tests for comparisons of before LSS, after $5 \mathrm{dyne} / \mathrm{cm}^{2}$, and after 20 dyne $/ \mathrm{cm}^{2}$ LSS. Two-way ANOVA was computed to examine any race by shear stress interaction effects. 
Densitometric analyses were completed using the ImageJ software (NIH Freeware) to quantify protein expression levels. All protein measurements were normalized to the $\beta$-actin internal control expression, and these normalized values were used for all statistical analyses.

All statistical analyses were performed using Microsoft Excel and SPSS version 18.0 (SPSS Inc., Chicago, IL). Data are expressed as mean \pm SE and the level of significance set at $P<0.05$.

\section{Results \\ Differential response in NADPH oxidase in response to magnitude of LSS}

Figure 1 shows a representative band showing the NADPH oxidase subunit protein expression levels by race with LSS. There was a significant racial difference under static conditions for all three subunits; p47phox $(P=0.04)$, NOX2 $(P=0.04)$, and NOX4 $(P=0.001)$ where the African American HUVECs had higher protein expression levels in each case. The only significant LSS effect on p47phox protein expression was in African American HUVECs. Relative to static controls, $\mathrm{p} 47 \mathrm{phox}$ protein expression levels significantly decreased with 20 dyne $/ \mathrm{cm}^{2} \operatorname{LSS}(P=0.046)$. LSS had no effect on NOX2 protein expression in HUVECs from either race.

Relative to static controls, NOX4 protein expression only decreased significantly in the African American HUVECs $(P=0.045)$ with 5 dyne $/ \mathrm{cm}^{2}$ LSS. But with 20 dyne $/ \mathrm{cm}^{2}$ LSS, it significantly decreased in both African American and Caucasian HUVECs ( $P=0.03$ and $P=0.04$, respectively). Thus, overall LSS had a larger effect on the NADPH subunit expressions in the African American HUVECs.

\section{NO production has a dose- dependent increase}

Figure 2 shows the eNOS protein expression and NO endproduct levels by HUVEC race with LSS. There was a significant racial difference $(P=0.04)$ under static conditions, with African American HUVECs having higher eNOS protein levels. In response to LSS, eNOS expression was
A

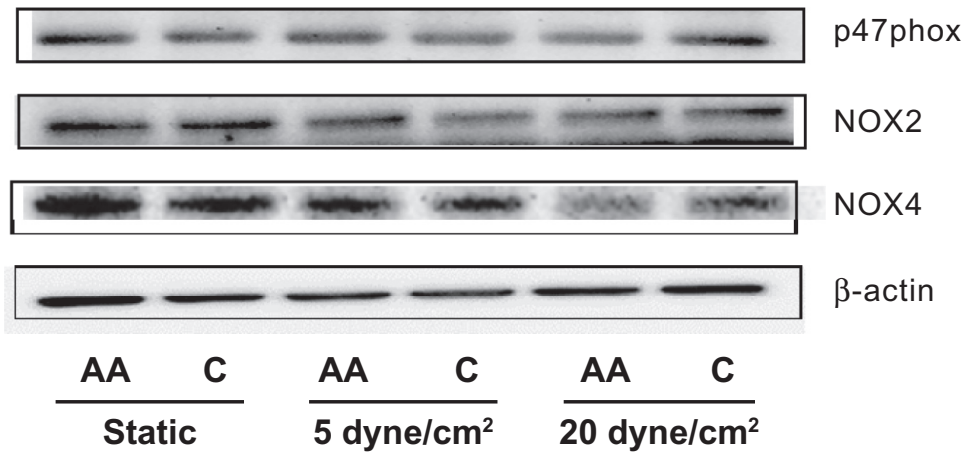

B

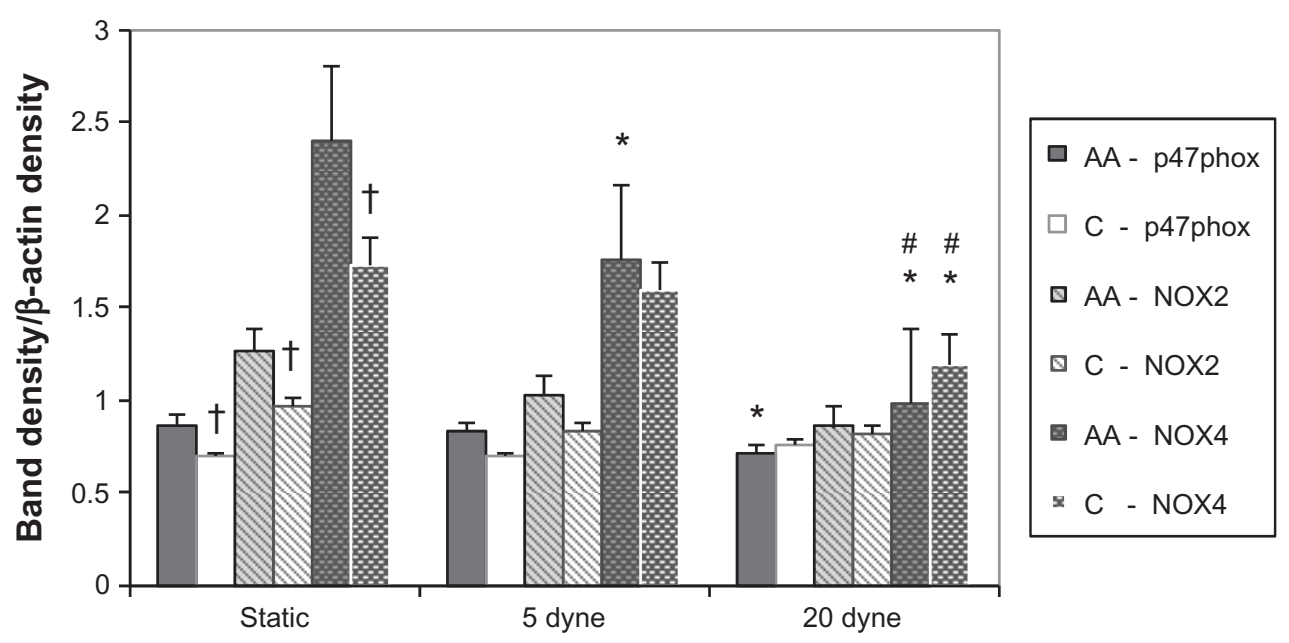

Figure I NADPH oxidase protein expression levels by race with laminar shear stress. (A) Representative western blots and $\beta$-actin control blot for African American (AA) and Caucasian (C) human umbilical vein endothelial cells; (B) ImageJ densitometric analysis of bands expressed in relation to $\beta$-actin.

Notes: Bars show mean \pm SE. $* P<0.05$ from static, within group; ${ }^{\#} P<0.05$ from 5 dyne $/ \mathrm{cm}^{2}$, within group; ${ }^{\dagger} P<0.05$ between ethnic groups. 
A
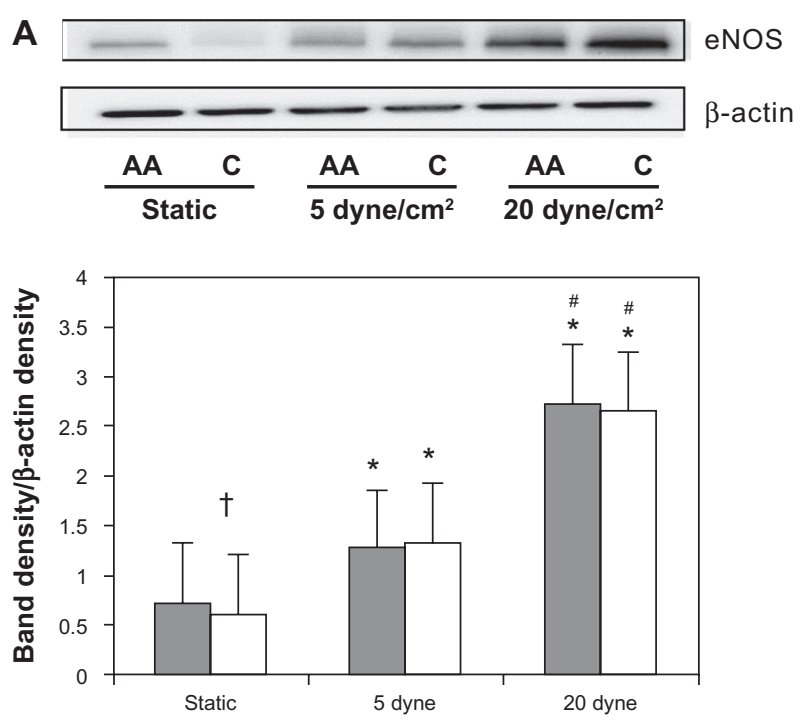

B

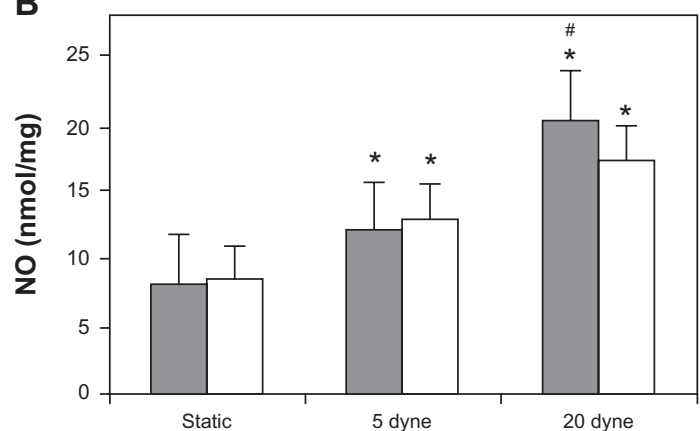

Figure 2 Endothelial inducible nitric oxidase synthase (eNOS) protein expression and $\mathrm{NO}$ end-product production by race with laminar shear stress. (A) Representative Western blot and $\beta$-actin control blot for African American (AA) and Caucasian (C) human umbilical vein endothelial cells (HUVECs). Imagej densitometric analysis of bands expressed in relation to $\beta$-actin; (B) total $\mathrm{NO}$ end-product production with LSS application in African American (solid bars) and Caucasian (open bars) HUVEC cell culture supernatant.

Notes: Bars show mean \pm SE. $* P<0.05$ from static; ${ }^{*} P<0.05$ from 5 dyne/ $\mathrm{cm}^{2}$; ${ }^{\dagger} P<0.05$ between ethnic groups.

significantly increased in both African American and Caucasian HUVECs at 5 dyne $/ \mathrm{cm}^{2}(P=0.001$ and $P=0.01$, respectively) and at 20 dyne $/ \mathrm{cm}^{2}(P=0.00$ and $P=0.002$, respectively). Also, relative to eNOS at 5 dyne $/ \mathrm{cm}^{2}$, expression significantly increased in both African American and Caucasian HUVECs with LSS at 20 dyne $/ \mathrm{cm}^{2}(P=0.0002$ and $P=0.001$, respectively).

Relative to static controls, HUVEC from both African American and Caucasians increased total $\mathrm{NO}$ production when exposed to LSS at both 5 dyne $/ \mathrm{cm}^{2}(P=0.001$ and $P=0.01$ respectively) and at 20 dyne $/ \mathrm{cm}^{2}(P=0.00$ and $P=0.02$, respectively). However, compared to the $5 \mathrm{dyne} / \mathrm{cm}^{2}$ condition, NO production at 20 dyne $/ \mathrm{cm}^{2}$ increased significantly only in African American HUVECs $(P=0.0003)$. There were no differences between HUVEC races in any of the conditions for eNOS protein expression or NO end-product production.

\section{Analysis of antioxidant responses to magnitude of LSS}

Figure 3 shows the antioxidant protein expressions and activity by race with LSS. In response to LSS, Catalase expression was significantly increased in both African American and Caucasian HUVECs at 5 dyne $/ \mathrm{cm}^{2}(P=0.004$ and $P=0.02$, respectively). Although catalase decreased in HUVECs from both races when exposed to LSS at $20 \mathrm{dyne} / \mathrm{cm}^{2}$ relative to LSS at 5 dyne $/ \mathrm{cm}^{2}$, only the African American HUVECs had a significant reduction $(P=0.01)$. Despite this, relative to static controls, catalase expression tended to increase with LSS at 20 dyne $/ \mathrm{cm}^{2}$ in HUVECs from both races.

Similar trends were observed in SOD2 expression (Figure 3B). There was a significant LSS and race interaction effect $(P=0.018)$. Also, under static conditions there was a significant racial difference where the Caucasian HUVECs had higher SOD2 expression $(P=0.01)$. In response to LSS, SOD2 protein expression increased significantly in African American HUVECs with magnitudes of both 5 dyne $/ \mathrm{cm}^{2}$ $(P=0.002)$ and 20 dyne $/ \mathrm{cm}^{2}(P=0.04)$.

Confirming this result, in cell lysate there was a significant racial difference in total SOD activity under static conditions, the Caucasian HUVECs having higher total SOD activity ( $P=0.004$, Figure $3 \mathrm{C})$. Again, in response to LSS, only the African American HUVECs had a significant increase in total SOD activity with both 5 dyne $/ \mathrm{cm}^{2}$ and 20 dyne $/ \mathrm{cm}^{2}(P=0.02$ and $P=0.003$, respectively) compared with static conditions. LSS had no effect on either SOD2 protein expression or total SOD activity in the Caucasian HUVECs, suggesting a larger SOD antioxidant response to LSS in African American HUVECs.

Finally, to obtain a representative measure of the sum total antioxidant capacity levels, TAC was measured in cell lysates (Figure 3D). There was only a significant racial difference between groups under the 5 dyne $/ \mathrm{cm}^{2}$ condition, the African American HUVECs having higher TAC levels $(P=0.02)$. Compared with static conditions, the African American HUVECs had more responses to application of LSS than the Caucasian HUVECs: a significant higher TAC level with LSS at both 5 dyne $/ \mathrm{cm}^{2}$ and 20 dyne $/ \mathrm{cm}^{2}(P=0.01$ and $P=0.03$, respectively) was observed.

\section{Discussion}

The main findings of this initial observatory study were that, overall, the African American HUVECs were more responsive to LSS than Caucasian HUVECs as evidenced by significant increases in antioxidant production and decreases in NADPH oxidase isoform expressions. In the present 
A
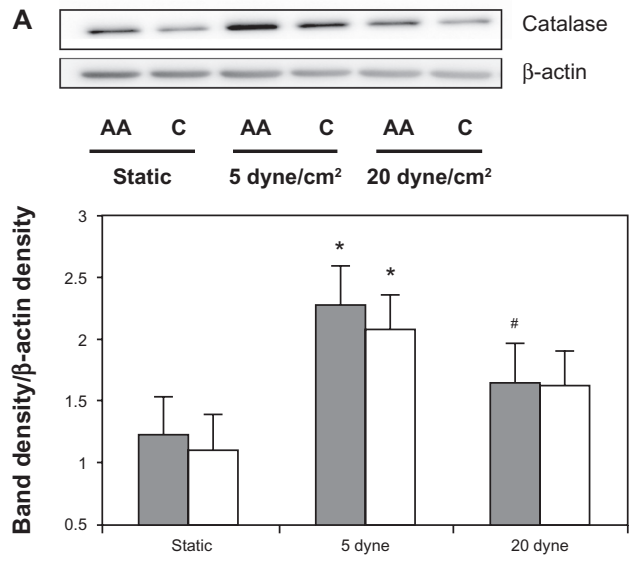

B

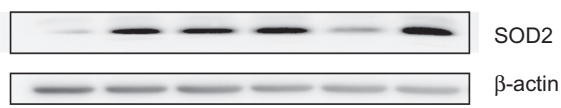

$\frac{\text { AA C }}{\text { Static }} \frac{\text { AA C }}{5 \text { dyne } / \mathrm{cm}^{2}} \frac{\text { AA C C }}{20 \text { dyne } / \mathrm{cm}^{2}}$
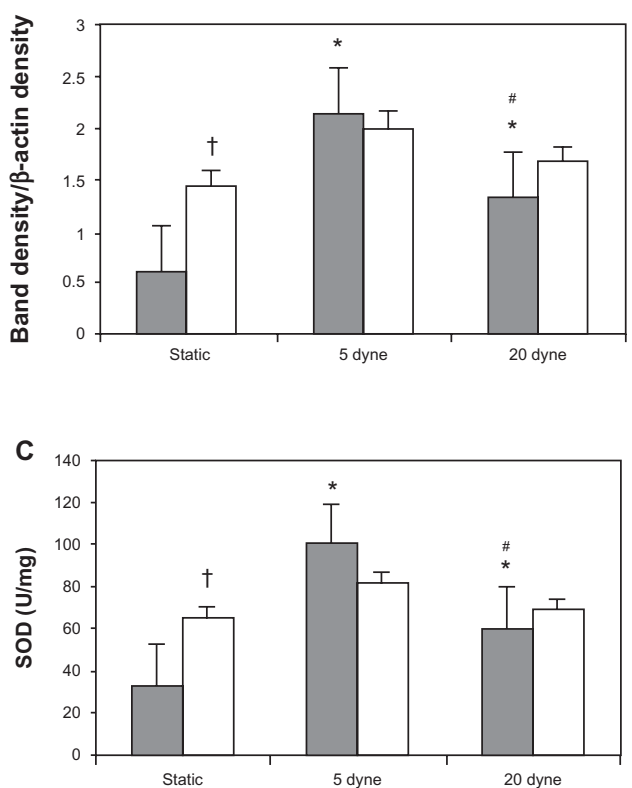

D

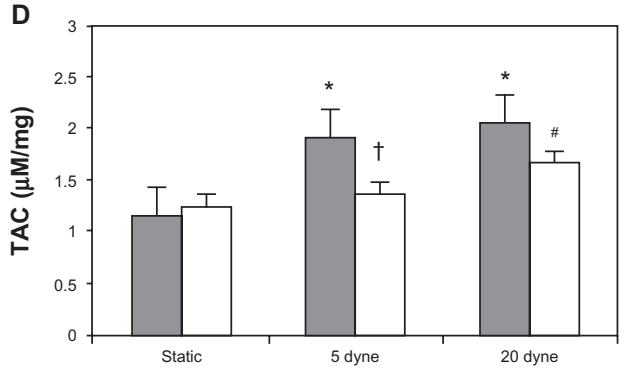

Figure 3 Antioxidant protein expression and activity by race with laminar shear stress. (A) Catalase representative Western blots and $\beta$-actin control blot for African American (AA) and Caucasian (C) human umbilical vein endothelial cells (HUVECs). ImageJ densitometric analysis of bands expressed in relation to $\beta$-actin; (B) representative superoxide dismutase 2 (SOD2) protein expression and $\beta$-actin control; (C) total SOD activity; (D) total antioxidant activity (TAC) levels with laminar shear stress application in African American (solid bars) and Caucasian (open bars) HUVEC cell lysate.

Notes: Bars show mean \pm SE. $* P<0.05$ from static; $\# P<0.05$ from 5 dyne $/ \mathrm{cm}^{2}$; $+P<0.05$ between ethnic groups. study, endothelial cell oxidative stress was measured by NO production, antioxidant capacity, and potential for superoxide $\left(\mathrm{O}_{2}^{-}\right)$production through NADPH oxidase subunit protein expressions. Overproduction of ROS leads to uncoupling of the eNOS enzyme, reduced NO production or bioavailability, and impaired antioxidant defenses, which are all associated with endothelial dysfunction. The central hypothesis examined by this study was whether at the cellular level there are racial differences in response to increased fluid flow using in vitro shear stress as an exercise mimetic. This was based on the premise that increased blood flow from aerobic exercise ameliorates endothelial dysfunction by creating a net antiatherogenic and antioxidant endothelial cell phenotype.

Through the years, the effects of both acute and chronic shear stress on endothelial cells in vitro have been extensively studied. In vivo, acute shear stress effects can be observed only when new flow is initiated (such as those through newly formed vessels) or when repair to an injured vessel wall occurs. Therefore, it can also be hypothesized that acute effects are seen in vivo in vascular beds of unconditioned individuals when a new aerobic exercise training program is initiated and the vessels are exposed to increased blood flow, thus highlighting the importance of the present study.

The results from this study confirm that application of LSS to HUVECs decreased potential oxidative stress levels, increased antioxidant activity, and increased NO production. All of these adaptations are indications of improved endothelial function and have separately been shown in vivo to have association with aerobic exercise adaptations. Therefore, the results presented here provide valuable evidence suggesting that this in vitro model of aerobic exercise, using shear stress to represent the increased blood flow, may be an effective tool for studying aerobic exercise adaptations of the endothelial cell layer.

Furthermore, the data from this study confirm the wellestablished in vitro upregulation of eNOS and increase in NO production that occurs with LSS. This adaptation was seen in HUVECs from both races. Interestingly, the observed racial difference in eNOS protein expression level that existed in the HUVECs, under static conditions, was not present after the application of LSS.

Similarly, in all of the measured NADPH oxidase subunits, a significant racial difference existed under static conditions. We found that African American HUVECs had higher p47phox, NOX2, and NOX4 protein expression levels. To the best of our knowledge, no study has shown racial differences in NOX2 or NOX4 expressions. Recent studies have purported that NOX4 and NOX2 are the primary NADPH 
oxidase homologs in the endothelium and thus are the only homologs studied in this current study. Again, after application of LSS the racial differences under static conditions no longer existed. Limited studies have specifically examined exercise training effects on NADPH oxidase activity and reported that exercise reduces the expression of NADPH oxidase and thereby decreases oxidative stress. ${ }^{10}$ Duerrschmidt et al used cultured HUVECs to quantify the expression levels of specific NADPH subunits in response to LSS and showed reduced mRNA and protein expression of NADPH oxidase subunits gp91phox (NOX2) and p47phox after 24 hours of LSS at 30 dyne $/ \mathrm{cm}^{2}$, a higher magnitude than was used in the present study. ${ }^{11}$ We found that African American HUVECs had a significant decrease in $\mathrm{p} 47 \mathrm{phox}$ protein expression with LSS at 20 dyne $/ \mathrm{cm}^{2}$, a significant dose-response decrease in NOX4 protein expression with LSS at 5 and $20 \mathrm{dyne} / \mathrm{cm}^{2}$, while the Caucasian HUVECS demonstrated a significant decrease in NOX4 expression only with $20 \mathrm{dyne} / \mathrm{cm}^{2}$. No study has measured NOX4 protein expression after LSS at 5 or 20 dyne/ $\mathrm{cm}^{2}$ in HUVECs, and therefore the present study is the first to report such findings and will thus need to be replicated by others. We also found that NOX2 protein expression was not changed by application of LSS at 5 or $20 \mathrm{dyne} / \mathrm{cm}^{2}$ suggesting that a higher magnitude may be necessary to elicit reductions similar to that seen by Duerrschmidt et al with LSS at 30 dyne $/ \mathrm{cm}^{2}$. Further research is needed to examine endothelial cell responses to LSS at different magnitudes in order to better understand and associate in vitro LSS with the adaptations caused by aerobic exercise blood flows.

It was in the 1960s and 1970s when engineering studies began to elucidate actual blood flow velocities. ${ }^{12-14}$ Since then, decades of molecular research have continued to study the in vitro effects of varying magnitudes of shear stress. However, few studies have actually studied blood flow during exercise. It has been shown that during moderate exercise the shear stress increases to between 7 and $50 \mathrm{dyne} / \mathrm{cm}^{2}$, blood flow shear levels depending on vessel type, size, and location. ${ }^{15-18}$ It is possible that endothelial cells located in different spatial regions of the vasculature are subjected to different levels of shear stress and thus may have different biological and biochemical adaptations. Therefore the continued research on the effects of LSS at different magnitudes is paramount.

We found that relative to static controls, LSS at $5 \mathrm{dyne} / \mathrm{cm}^{2}$ for 24 hours differentially regulated protein expression. The NADPH oxidase subunits p47phox and NOX2 were not affected by this level of LSS, while NOX4 expression decreased in the African American HUVECs. These findings confirm previous findings. Data from Duerrschmidt et al showed that 24 hours of LSS at $5 \mathrm{dyne} / \mathrm{cm}^{2}$ had no effect on p47phox or NOX2 expression. ${ }^{11}$ Likewise, De Keulenaer et al measured intracellular $\mathrm{O}_{2}^{-}$production and showed that overall $\mathrm{O}_{2}^{-}$levels in HUVECs exposed to 24 hours of LSS at 5 dyne $/ \mathrm{cm}^{2}$ had no difference relative to static control cells. ${ }^{19}$ Furthermore, the results of their study also showed that steady LSS at 5 dyne $/ \mathrm{cm}^{2}$ caused a concomitant increase in CuZnSOD protein expression. We also found that all of the antioxidant enzymes increased with LSS at $5 \mathrm{dyne} / \mathrm{cm}^{2}$. These data indicate that another source of $\mathrm{O}_{2}^{-}$may be activated at this low level of LSS.

To summarize, these data for the first time provide cellular evidence that supports the prescription of exercise by physicians as a nonpharmacological intervention aimed at improving vascular function, because we show that application of LSS to HUVECs decreased oxidative stress levels, increased antioxidant activity, and increased NO production. Our data also suggest that it could be hypothesized that African Americans may have a larger improvement in endothelial function from the increased blood flow that accompanies aerobic exercise training, compared with Caucasians. Within the scope of this study, mechanisms underlying the observed response were not studied, only whether racial differences existed. Also it is recognized that venous and arterial endothelial cells are not identical, but is it known that all of the genes associated with the markers measured in the present study are expressed in both veins and arteries. So, using the results of this observational study, future molecular research should continue to investigate endothelial cell responses to varying magnitudes of LSS and also could aim to identify specific mechanisms or transducers responsible for the racial differences observed herein. With respect to shear stress, considerable attention is currently focused on the fundamental question of the identity, function, and mechanisms of actions of the endothelial flow-mediated mechanotransducers. From the results of the present study, it is clear that such research could also now focus on identifying race-dependent associations in mechanotransduction so as to identify specific therapeutics for endothelial dysfunction, since we have shown that African American and Caucasian HUVECs may have different regulation.

\section{Acknowledgments}

This research was supported by NIH/NHLBI Grant RO1 HL085497 (PI, Michael Brown) and by NIH/NIA Grant KO1AG019640 (PI, Michael Brown). 


\section{Disclosure}

The authors report no conflicts of interest.

\section{References}

1. Hozawa A, Folsom AR, Sharrett AR, Chambless LE. Absolute and attributable risks of cardiovascular disease incidence in relation to optimal and borderline risk factors: comparison of African American with white subjects - atherosclerosis risk in communities study. Arch Intern Med. 2007; 167:573-579.

2. Carroll JF, Fulda KG, Chiapa AL, et al. Impact of race/ethnicity on the relationship between visceral fat and inflammatory biomarkers. Obesity (Silver Spring). 2009;17:1420-1427.

3. Vaziri ND, Rodriguez-Iturbe B. Mechanisms of disease: oxidative stress and inflammation in the pathogenesis of hypertension. Nat Clin Pract Nephrol. 2006;2:582-593.

4. Chen X, Andresen BT, Hill M, Zhang J, Booth F, Zhang C. Role of reactive oxygen species in tumor necrosis factor-alpha induced endothelial dysfunction. Curr Hypertens Rev. 2008;4:245-255.

5. Csanyi G, Taylor WR, Pagano PJ. NOX and inflammation in the vascular adventitia. Free Radic Biol Med. 2009;47:1254-1266.

6. Feairheller DL, Park J-Y, Sturgeon KM, Williamson S, Diaz KM, Veerabhadrappa P, Brown MD. Racial differences in oxidative stress and inflammation: in vitro and in vivo. Clin Transl Sci. 2011;4:32-37.

7. Gooch KJ, Frangos JA. Flow- and bradykinin-induced nitric oxide production by endothelial cells is independent of membrane potential. Am J Physiol. 1996;270:C546-C551.

8. Mowbray AL, Kang DH, Rhee SG, Kang SW, Jo H. Laminar shear stress up-regulates peroxiredoxins (PRX) in endothelial cells: PRX 1 as a mechanosensitive antioxidant. J Biol Chem. 2008;283:1622-1627.

9. Feairheller DL, Sturgeon KM, Diaz KM, et al. Prehypertensive AfricanAmerican women have preserved nitric oxide and renal function but high cardiovascular risk. Kidney Blood Press Res. 2010;33:282-290.
10. Adams V, Linke A, Krankel N, et al. Impact of regular physical activity on the $\mathrm{NAD}(\mathrm{P}) \mathrm{H}$ oxidase and angiotensin receptor system in patients with coronary artery disease. Circulation. 2005;111:555-562.

11. Duerrschmidt N, Stielow C, Muller G, Pagano PJ, Morawietz H. $\mathrm{NO}-$ mediated regulation of $\mathrm{NAD}(\mathrm{P}) \mathrm{H}$ oxidase by laminar shear stress in human endothelial cells. $J$ Physiol. 2006;576:557-567.

12. Cohen A, Gallagher JP, Luebs ED, et al. The quantitative determination of coronary flow with a positron (rubidium-84). Circulation. 1965; 32:636-649

13. Folts JD, Rowe GG, Kahn DR, Young WP. Phasic changes in human right coronary blood flow before and after repair of aortic insufficiency. Am Heart J. 1979;97:211-215.

14. Lipowsky HH, Kovalcheck S, Zweifach BW. The distribution of blood rheological parameters in the microvasculature of cat mesentery. Circ Res. 1978;43:738-749.

15. Tang BT, Cheng CP, Draney MT, et al. Abdominal aortic hemodynamics in young healthy adults at rest and during lower limb exercise: quantification using image-based computer modeling. Am J Physiol Heart Circ Physiol. 2006;291:H668-H676.

16. Cheng C, Helderman F, Tempel D, et al. Large variations in absolute wall shear stress levels within one species and between species. Atherosclerosis. 2007;195:225-235.

17. Draney MT, Herfkens RJ, Hughes TJ, et al. Quantification of vessel wall cyclic strain using cine phase contrast magnetic resonance imaging. Ann Biomed Eng. 2002;30:1033-1045.

18. Tang BT, Fonte TA, Chan FP, Tsao PS, Feinstein JA, Taylor CA. Three-dimensional hemodynamics in the human pulmonary arteries under resting and exercise conditions. Ann Biomed Eng. 2011;39: $347-358$.

19. De Keulenaer GW, Chappell DC, Ishizaka N, Nerem RM, Alexander RW, Griendling KK. Oscillatory and steady laminar shear stress differentially affect human endothelial redox state: role of a superoxide-producing NADH oxidase. Circ Res. 1998;82:1094-1101.
Vascular Health and Risk Management

\section{Publish your work in this journal}

Vascular Health and Risk Management is an international, peerreviewed journal of therapeutics and risk management, focusing on concise rapid reporting of clinical studies on the processes involved in the maintenance of vascular health; the monitoring, prevention and treatment of vascular disease and its sequelae; and the involvement of

\section{Dovepress}

metabolic disorders, particularly diabetes. This journal is indexed on PubMed Central and MedLine. The manuscript management system is completely online and includes a very quick and fair peer-review system, which is all easy to use. Visit http://www.dovepress.com/ testimonials.php to read real quotes from published authors. 